\title{
Investigation on the Optical Properties of Nonlinear Optical (NLO) Single Crystal: L-Valine zinc hydrochloride
}

\author{
Suresh Sagadevan \\ Department of Physics, Sree Sastha Institute of Engineering and Technology, Chennai-123
}

Email address:

sureshsagadevan@gmail.com

\section{To cite this article:}

Suresh Sagadevan. Investigation on the Optical Properties of Nonlinear Optical (NLO) Single Crystal: L-Valine Zinc Hydrochloride. American Journal of Optics and Photonics. Vol. 2, No. 3, 2014, pp. 24-27. doi: 10.11648/j.ajop.20140203.11

\begin{abstract}
The development and encroachment of high technology, from transportation, computation to information is based on the availability of materials in the form of single crystals. With progress in crystal growth technology and characterization, organic, inorganic, semiorganic and organometallic materials having attractive nonlinear optical (NLO) properties are being discovered at rapid rate. NLO materials possess several attractive properties such as high NLO coefficient, high laser damage threshold, wide transparency range, high mechanical strength and thermal stability, which make the materials suitable for second harmonic generation (SHG) and other NLO applications. Single crystals of L-Valine zinc hydrochloride were grown by the slow evaporation technique. The single crystal X-ray diffraction analysis reveals that the crystal belongs to the monoclinic system. The optical absorption spectrum reveals the transparency of the crystal in the entire visible region and the cut off wave length has been found to be $210 \mathrm{~nm}$. The optical band gap is found to be $5.91 \mathrm{eV}$. Optical constants such as the band gap $\left(E_{g}\right)$, refractive index $(n)$, reflectance $(R)$, extinction coefficient $(\mathrm{K})$ and the real $\left(\varepsilon_{r}\right)$ and imaginary $\left(\varepsilon_{i}\right)$ components of the dielectric constant and electric susceptibility $\left(\chi_{c}\right)$ were determined from the UV-VISNIR absorption spectrum.
\end{abstract}

Keywords: Optical Transmission Spectrum, Optical band gap, Refractive Index (n), Reflectance (R), Extinction Coefficient (K)

\section{Introduction}

One of the important applications in photonic technology is the use of nonlinear optical (NLO) effects. A nonlinear optical effect is the interaction of an electromagnetic field of high intensity laser light with a material. Materials with large nonlinear optical susceptibilities are of importance in optoelectronics, where they are used in the construction of the optical analogues of the circuit elements-modulators, amplifiers, rectifiers, switches, and so forth, of conventional electronics. Due to high-speed and ease of production of photons (light), the area of photonics has become an active field of research in view of the modern society's demand for improved telecommunications, data storage, retrieving, processing and transmission. The design of devices that utilize photons instead of electrons for the transmission of information has created a need for new materials with unique nonlinear optical (NLO) materials. The nonlinear optics is the field which includes all phenomena in which optical parameters of materials are changed with the interaction of intense coherent source of light. The NLO phenomena have lead to the enhancement in understanding of light-matter interactions. The search for new molecular materials with NLO properties is currently the subject of considerable importance investigations due to their potential applications in photonic devices [1]. Nonlinear optics (NLO) is an innovative area of research and development which will play a key role in the field of optoelectronics and photonics [2]. The apparent development of semiorganic materials, where the organic ligand is ionically bonded with inorganic host refined the search of new materials with high optical nonlinearities which is an important area due to their optical applications such as optical communication, optical computing, optical information processing, optical disk data storage, laser fusion reaction, laser remote sensing, colour display, medical diagnostics, etc. [3]. Nonlinear optical (NLO) materials play a major role in nonlinear optics and in particular they have a great impact on information technology and industrial applications [4]. In the present investigation, report the optical properties of the L-valine zinc hydrochloride single crystal. 


\section{Materials and Methods}

The L-valine zinc hydrochloride single crystal was synthesized from L-valine, zinc sulphate heptahydrate, and hydrochloric acid. The solution was agitated with a magnetic stirring device for $3 \mathrm{~h}$ continuously and filtered after the complete dissolution of the starting materials. The obtained salt was filtered and dried at room temperature. The solution thus prepared was allowed to evaporate at room temperature and crystals of good optical qualities have been harvested from the solution in 30 days. Single crystals were extracted and subjected to single crystal XRD studies. Single crystal X-ray diffraction (X-ray crystallography) is an analytical technique in which X-rays are employed to determine the actual arrangement of atoms within a crystalline specimen. Single crystal X-ray diffraction is a non-destructive tool to analyze crystal structure of compounds, which can be grown as single crystals. The molecular structure, atomic coordinates, bond lengths, bond angles, molecular orientation and packing of molecules in single crystals can be determined by X-ray crystallography. Single crystal X-ray diffractometer collects intensity data required for structure determination. In the present study, the single crystal X-ray diffraction analysis was performed using an ENRAF NONIUS CAD4-F single crystal X-ray diffractometer. Single crystal X-ray diffraction analysis was carried out to determine the lattice parameters. The unit cell parameters were determined using the single crystal X-ray diffraction. The unit cell parameters are $\mathrm{a}=9.44 \AA, \mathrm{b}=5.40 \AA, \mathrm{c}=11.84 \AA$ and $\alpha=\gamma=90^{\circ}$, $\beta=92.66^{\circ}$. From the XRD, it is observed that the grown crystal belongs to the monoclinic system with noncentrosymmetric space group $\mathrm{P} 2{ }_{1}$. The absorption spectrum of L-valine zinc hydrochloride was recorded with a Varian Cary 5E spectrophotometer in the range of 200-1200 nm. UV-Vis-NIR spectroscopy might be defined as the measurement of the absorption or emission of radiation associated with changes in the spatial distribution of electrons in atoms and molecules. In practice, the electrons involved are usually the outer valence or bonding electrons, which can be excited by the absorption of UV or visible or near IR radiation.

\section{UV-Visible Spectroscopy}

Ultraviolet-visible spectroscopy (UV/ VIS) is also known as electronic spectroscopy. Ultraviolet (200 - 400 $\mathrm{nm}$ ) and visible (400 - $800 \mathrm{~nm}$ ) absorption spectroscopy is the measurement of the attenuation of a beam of light after it passes through a sample or after reflection from a sample surface. It uses light in the visible and adjacent near ultraviolet (UV) and near infrared (NIR) ranges. In this region of energy space molecules undergo electronic transitions. Ultraviolet and visible light are energetic enough to promote outer (valence) electrons to higher energy levels. Valence electrons are found in three types of electron orbitals namely $\sigma$ bonding orbitals, $\pi$ bonding orbitals and non-bonding orbitals (n-lone pair electrons). Sigma $(\sigma)$ bonding orbitals tend to be lower in energy than $\pi$ bonding orbitals, which in turn are lower in energy than non-bonding orbitals. The unoccupied or anti bonding orbitals $\left(\sigma^{*}\right.$ and $\left.\pi^{*}\right)$ are the orbitals of highest energy. An energy level diagram showing electronic transitions is depicted in Fig. 1 of the six transitions outlined, only the two lowest energy ones (left-most, coloured blue) are achieved by the energies available in the 200 to $800 \mathrm{~nm}$ spectrum. As a rule, energetically favored electron promotion will be from the highest occupied molecular orbital (HOMO) to the lowest unoccupied molecular orbital (LUMO) and the resulting species is called an excited state. The experimental arrangement of Varian Cary 5E UV-VisNIR Spectrophotometer is shown in Fig.2 UV-Vis-NIR spectroscopy might be defined as the measurement of the absorption or emission of radiation associated with changes in the spatial distribution of electrons in atoms and molecules. Electronic transitions are possible for a wide range of vibrational levels within the initial and final electronic states. Saturated hydrocarbons and compounds containing only alkyl groups, alcohol groups and ether groups are transparent in the region 200-1000 nm. Such compounds are useful as solvents for making solutions of the specimen to study in this region. An isolated functional group not in conjugation with any other group is said to be a chromophore if it exhibits absorption of a characteristic nature in the ultraviolet or visible region. If a series of compounds has the same functional group and no complicating factors are present, all of them will generally absorb at very nearly the same wavelength. Thus, it is readily seen that the spectrum of a compound, when correlated with data from the literature for known compounds, can be a very valuable aid in determining the functional groups present in the molecule.

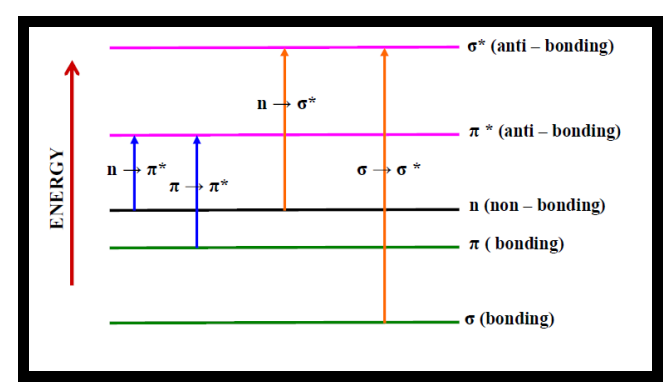

Fig. 1. Energy level diagrams with electronic transitions

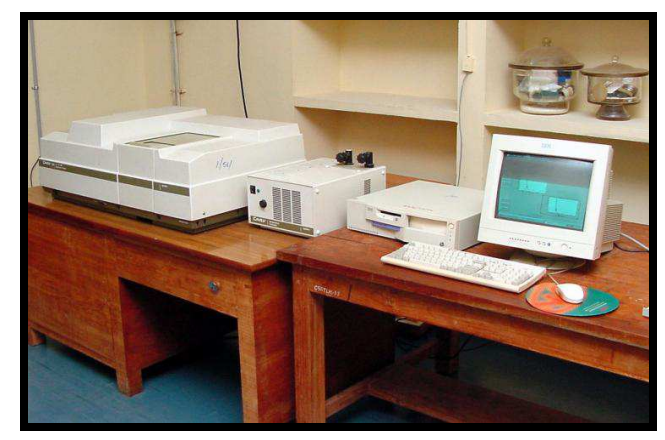

Fig.2. Experimental arrangement of Varian Cary $5 E$ UV-Vis-NIR Spectrophotometer 
In particular, absorption bands results from transitions ( $\pi \rightarrow \pi^{*}$ ) and $n \rightarrow \pi^{*}$ involving $\pi$-orbitals and lone pairs ( $\mathrm{n}=$ non-bonding) are important and so UV-Vis spectroscopy is of most use for identifying conjugated systems which tend to have stronger absorptions. Absorption bands can also arise from certain molecules where the energy required for removing an electron from one atom and placing it on another falls within the UV/Vis region. This process is known as a charge transfer excitation. Molecules with the ability to exhibit the above types of electronic transitions are said to possess chromophores. An isolated functional group not in conjugation with any other group is said to be a chromophore if it exhibits absorption of a characteristic nature in the ultraviolet or visible region. The most common are $(\mathrm{C}=\mathrm{C})$ and $(\mathrm{C}=\mathrm{O})$ which exhibits $\left(\pi \rightarrow \pi^{*}\right)$ and $n \rightarrow \pi *$ transitions respectively.

If a series of compounds have the same functional group and no complicating factors are present, all of them will generally absorb at very nearly the same wavelength. Thus, it is readily seen that the spectrum of a compound, when correlated with data from the literature for known compounds, can be a very valuable aid in determining the functional groups present in the molecule. Samples are typically placed in a transparent cell, known as a cuvette. The sample holders (cuvettes) are the rectangular shaped quartz or glass cells of about $10 \mathrm{~mm}$ path length. The transmitted light radiation is received at the photomultiplier tube alternately from the reference and the sample beams. A photoelectric signal timing system is synchronized with the alternate pulses which permits the comparison of signals from the two beams. The difference between the two signals is recorded with the help of a motor driven pen or is interfaced with a PC-XT and stored there for easy reference. Samples in solid form, powder, pellets are dissolved in suitable solvents to form the contents of the sample cell and the solvents are taken in the reference cell.

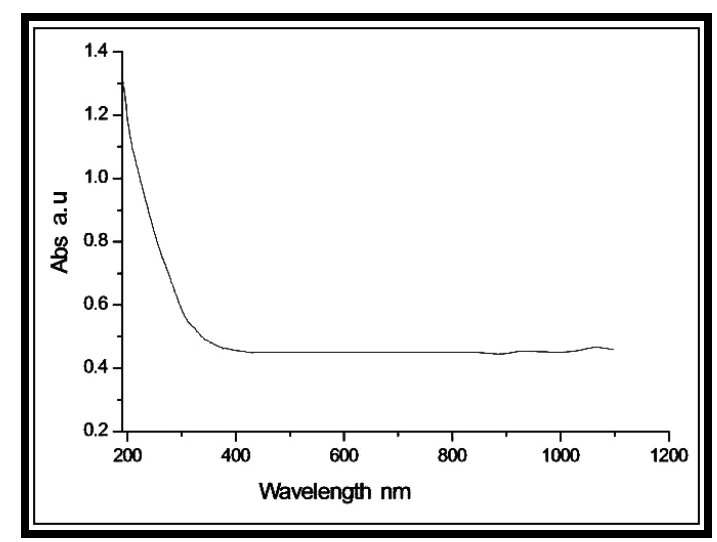

Fig. 3. Absorption spectrum of L-valine zinc hydrochloride crystal

In the present work, the UV-Visible spectrum was recorded using Varian Cary 5E UV-Vis-NIR Spectrophotometer. The spectrum gives information about the structure of the molecule because the absorption of UV and visible light involves promotion of the electron in the $\sigma$ and $\pi$ orbital from the ground state to higher states. The optical absorption spectrum of L-valine zinc hydrochloride crystal shown in Fig. 3 was recorded between 200 and 1200 $\mathrm{nm}$. The crystal has excellent transmission in the entire visible region. The lower cutoff wavelength is $210 \mathrm{~nm}$ single crystals are mainly used in optical applications and hence optical transmittance window and the transparency lower cut off ( $200 \mathrm{~nm}-400 \mathrm{~nm})$ is very important for the realization of SHG output in this range using lasers. This transparent nature in the visible region is a desirous property for the material used for NLO applications.

From the absorption spectrum, the lower cut-off wavelength is found to be $210 \mathrm{~nm}$ and the lower percentage absorption indicates that the crystal readily allows the transmission of the laser beam in the range between $210 \mathrm{~nm}$ and $1200 \mathrm{~nm}$. It shows that the grown crystal has a good transparency in UV, visible and near IR region indicating that it can be used for NLO applications. The band gap of the crystal was estimated using the relation (1) [5]

$$
E_{g}=\frac{1.243 \times 10^{3}}{\lambda_{\max }}
$$

The band gap value found to be $5.91 \mathrm{eV}$, which is typical of dielectric materials. This high value of band gap indicates that the grown crystal possesses dielectric behavior to induce polarization when powerful radiation is incident on the material. The absence of absorption bands in the visible region and the wide band gap of the grown crystal confirm to the suitability of the grown crystal for photonic and optical applications. The larger energy band gap shows that the defect concentration in the grown crystal is very low.

\section{Determination of Optical Constants}

The optical behaviour of a material is important to determine its usage in optoelectronic devices [6]. A knowledge of the optical constants of a material, such as the optical band gap and extinction coefficient is quite essential, to examine the material's potential optoelectronic applications [7]. Further, the optical properties may also be closely related to the material's atomic structure, electronic band structure and electrical properties. An accurate measurement of the optical constant can be easily performed on inorganic crystals. In such crystals, the photonic density of states and photonic mode spatial distribution can be tailored with great accuracy. The optical constants (n, K) are determined from the transmission (T) and reflection $(\mathrm{R})$ spectrum following relations [8]. The transmittance $(\mathrm{T})$ is given by

$$
T=\frac{(1-R)^{2} \exp (-\alpha t)}{1-R^{2} \exp (-2 \alpha t)}
$$

where $t$ is the thickness and $\alpha$ is related to the extinction 
coefficient $(\mathrm{K})$ is and determined by

$$
K=\frac{\lambda \alpha}{4 \pi}
$$

The refractive index (n) can be determined from the reflectance $(\mathrm{R})$ [9]

$$
R=\frac{(n-1)^{2}}{(n+1)^{2}}
$$

The internal efficiency of the device also depends on wavelength. Hence, by tailoring the wavelength, one can achieve the desired material for the fabrication of electrooptic and optoelectronic devices. The reflectance $(\mathrm{R})$ in terms of the absorption coefficient can be obtained from the above equation. Hence,

$$
R=\frac{\exp (-\alpha t) \pm \sqrt{\exp (-\alpha t) T-\exp (-3 \alpha t) T+\exp (-2 \alpha t) T^{2}}}{\exp (-\alpha t)+\exp (-2 \alpha t) T}
$$

The refractive index (n) can be determined from the reflectance data using the following equation,

$$
n=\frac{-(R+1) \pm 2 \sqrt{n R}}{R-1}
$$

From the optical constants, the electric susceptibility $\left(\chi_{c}\right)$ can be calculated according to the following relation [10]

$$
\varepsilon_{r}=\varepsilon_{0}+4 \pi \chi_{C}=n^{2}-k^{2}
$$

Hence,

$$
\chi_{C}=\frac{n^{2}-k^{2}-\varepsilon_{0}}{4 \pi}
$$

The value of the electric susceptibility $\chi_{C}$ is 0.1628 at $\lambda=1000 \mathrm{~nm}$. The real part dielectric constant $\mathcal{E}_{r}$ and imaginary part dielectric constant $\varepsilon_{i}$ can be calculated from the following relations [11],

$$
\varepsilon_{r}=n^{2}-k^{2} \& \varepsilon_{i}=2 n k
$$

The value of real $\mathcal{E}_{r}$ and imaginary $\mathcal{E}_{i}$ dielectric constants at $\lambda=1000 \mathrm{~nm}$ are 1.353 and $5.723 \times 10^{-5}$ respectively. From the calculated values of the real and imaginary constants, the functions of the dielectrics were also determined. The real and imaginary parts of the dielectric constant of the grown crystal were determined.
The lower value of the dielectric constant with a wide band gap of the L-valine zinc hydrochloride crystal suggests its suitability for optoelectronic devices.

\section{Conclusion}

Single crystals of the L-valine zinc hydrochloride were grown by the slow evaporation technique. The grown crystals were characterized by the single crystal XRD and it is confirmed that the crystal belongs to the monoclinic system with non-centrosymmetric space group $\mathrm{P} 2_{1}$. The optical constants, such as the optical band gap $\left(E_{g}\right)$, absorption coefficient $(\alpha)$, extinction coefficient $(K)$, refractive index $(n)$, electric susceptibility $\left(\chi_{c}\right)$ and the real and imaginary dielectric constants were calculated to analyse the optical property of the crystal.

\section{Acknowledgement}

The author thanks the Management and Principal of Sree Sastha Institute of Engineering and Technology, Chembarambakkam, Chennai -600123 for their encouragements throughout the work.

\section{References}

[1] Prasad, P.N., and Williams D.J., Introduction to Nonlinear Optical Effects in Molecules and Polymers. Wiley, New York, 1991

[2] Bright, K.C., and Freeda, T.H.,Growth and characterization of organometallic L-alanine cadmium chloride single crystal by slow evaporation technique. Physica B., 2010, 405 (18), $3857-3861$.

[3] Moitra, S., and Kar,T., Growth and characterization of Lvaline- anonlinear optical crystal.Cryst. Res. Technol., 2010, 45(1), $70-74$

[4] Moitra,S., Seth, S. K., and Kar, T.,Synthesis, crystal structure, characterization and DFT studies of L-valine Lvalinium hydrochloride.J.Cryst. Growth., 2010, 312, 19771982.

[5] Sagadevan, S. and Murugasen, P. (2014) Journal of Crystallization Process and Technology, 4, 99-110 (2014)

[6] V. Pandey, N. Mehta, S.K. Tripathi, A. Kumar, Chalcogenide Lett., 2,39(2005)

[7] M. Dongol, Egypt. J. Solids, 25, 33(2002)

[8] S.Suresh and D.Arivuoli, Journal of Optoelectronics and Biomedical Materials. 3, 63 - 68 (2011)

[9] Sagadevan Suresh, Optik - International Journal for Light and Electron Optics 125, 950-953 (2014)

[10] Sagadevan Suresh, Optik - International Journal for Light and Electron Optics 125, 1223-1226(2014)

[11] Suresh Sagadevan, American Chemical Science journal, 3(3), 325-337, 2013 